\title{
Modeling of C stars with core/mantle grains: Amorphous carbon $+\mathrm{SiC}$
}

\author{
S. Lorenz-Martins ${ }^{1}$, F. X. de Araújo ${ }^{2}$, S. J. Codina Landaberry ${ }^{2}$, W. G. de Almeida² ${ }^{2}$ and R. V. de Nader ${ }^{1}$ \\ 1 Observatorio do Valongo/UFRJ, Ladeira Pedro Antonio 43, 20080-090, Rio de Janeiro, Brazil \\ 2 Observatorio Nacional/MCT, Rua Gal. Jose Cristino 77, 20921-400, Rio de Janeiro, Brazil
}

Received 4 August 2000 / Accepted 9 November 2000

\begin{abstract}
A set of 45 dust envelopes of carbon stars has been modeled. Among them, 34 were selected according to their dust envelope class (as suggested by Sloan et al. 1998) and 11 are extreme carbon stars. The models were performed using a code that describes the radiative transfer in dust envelopes considering core/mantle grains composed by an $\alpha$-SiC core and an amorphous carbon (A.C.) mantle. In addition, we have also computed models with a code that considers two kinds of grains $-\alpha$-SiC and A.C. - simultaneously. Core-mantle grains seem to fit dust envelopes of evolved carbon stars, while two homogeneous grains are more able to reproduce thinner dust envelopes. Our results suggest that there exists an evolution of dust grains in the carbon star sequence. In the beginning of the sequence, grains are mainly composed of $\mathrm{SiC}$ and amorphous carbon; with dust envelope evolution, carbon grains are coated in SiC. This phenomena could perhaps explain the small quantity of SiC grains observed in the interstellar medium. However, in this work we consider only $\alpha$-SiC grains, and the inclusion of $\beta$-SiC grains can perhaps change some of these results.
\end{abstract}

Key words. stars: carbon - circumstellar matter - radiative transfer

\section{Introduction}

Asymptotic giant branch (AGB) stars are often surrounded by circumstellar dust shells. The chemical composition of these media reflects that of the stellar photosphere. Thus, carbon-rich grains, such as amorphous carbon (A.C.), are one of the expected components of the dust envelopes around carbon stars. In addition, almost all these stars show an emission feature around $11.3 \mu \mathrm{m}$ due to Silicon carbide $(\mathrm{SiC})$ grains which are also condensed there. The existence of $\mathrm{SiC}$ grains in the atmospheres of carbon stars was predicted at first on the basis of chemical equilibrium calculations by Gilman (1969). This prediction was supported by the observations of Hackwell (1972), Treffers \& Cohen (1974) and Forrest et al. (1975). Nowadays infrared satellites provide several characteristic features of these grains.

After the observations with IRAS satellite, several works have dealt with the classification of dust envelopes around carbon stars. Little-Marenin et al. (1987) have discovered 176 new carbon stars using the feature at $11.3 \mu \mathrm{m}$ as a selection factor. Willems (1987) has analyzed 304 such objects, suggesting a SiC-index to the stars which present the emission feature varying between

Send offprint requests to: S. Lorenz-Martins, e-mail: lorenz@sun1.ov.ufrj.br
11.2-11.6 $\mu \mathrm{m}$. Papoular (1988) has classified carbon dust envelopes using the $11.3 \mu \mathrm{m}$ feature and a secondary feature at $8.6 \mu \mathrm{m}$. The sample of Chan \& Kwok (1990) was composed of 356 objects which were classified in two distinct classes. According to the authors, the difference between the classes is due to an evolution of $\alpha-\mathrm{SiC}$ and $\beta$ $\mathrm{SiC}$ particles. More recently Sloan et al. (1998, hereafter SLMP) proposed a classification of 89 carbon-rich stars in 6 different types based on their infrared emissions. All stars of their sample show the $\mathrm{SiC}$ feature at $11.3 \mu \mathrm{m}$.

In general, differences between each class can be interpreted as an evolution of the dust envelope itself due to an increasing amount of grains; consequently, optical depth also increases, affecting radiative transfer and the emission feature. On the other hand, the resonance features of small $\mathrm{SiC}$ grains are very sensitive to size, morphology and chemical composition of impurities in the surrounding medium (Bohen \& Huffman 1998). This fact suggests that the variety of emission features assigned to $\mathrm{SiC}$ grains is probably related to the formation process of the dust in circumstellar envelopes, reflecting the physical and chemical conditions. The classification criteria cited above are useful, but if one is interested in a deeper insight into the nature of the circumstellar dust grains, it is necessary to solve the radiative transfer problem, and to reproduce the 
features seen in the mid-IR LRS together with the overall behavior of the spectral energy distribution.

Several authors have calculated the radiative transfer in circumstellar dust shells (CDS) of carbon stars (Chan \& Kwok 1990; Lorenz-Martins \& Lefèvre 1993, 1994; Groenewegen 1995; Bagnulo, 1996; Bagnulo et al. 1998). Lorenz-Martins \& Lefèvre (1994) have employed the Monte Carlo method for solving the radiative transfer for two species simultaneously. SiC grains were supposed to form closer to the star than graphite grains (McCabe 1982). Correlations between $\mathrm{SiC} / \mathrm{A}$.C. ratio and extinction opacity as well as $\mathrm{SiC} / \mathrm{A}$.C. ratio and period of luminosity were found. These correlations indicate that the quantity of $\mathrm{SiC}$ grains relative to amorphous carbon grains decreases with carbon star evolution. Mass loss from cool stars is the major source of refractory grains in the interstellar medium. Carbon stars provide carboneous material and extreme carbon stars, with their large mass-loss rate, are the main contributors. A study by Whittet et al. (1990) shows that there is a low fraction $(<5 \%)$ of $\mathrm{Si}$ in the form of $\mathrm{SiC}$ in the interstellar medium. The enrichment rate for a kind of grain depends on the composition of the dust envelopes of stars which have high mass-loss rate. Lorenz-Martins \& Lefèvre (1994) also suggested that $\mathrm{SiC}$ grains are the minor component in carbon star envelopes. In addition, stars which have low $\mathrm{SiC} / \mathrm{A}$.C. ratios $(0.01-0.06)$ have also high mass-loss rates $\left(1.510^{-4}\right.$ - IRC+10216 - $6.110^{-6} M_{\odot} / \mathrm{yr}-$ IRAS $\left.15194-5115\right)$. These results can perhaps explain the small quantity of $\mathrm{SiC}$ grains in the interstellar medium. Alternatively, the weakness of the $\mathrm{SiC}$ absorption could be due to the fact that $\mathrm{SiC}$ particles are embedded in thick carbon mantles. In fact, Kozasa et al. (1996) have shown that the nucleation of $\mathrm{SiC}$ precedes that of carbon grains and may lead to the formation of dust grains consisting of a $\mathrm{SiC}$ core and a carbon mantle. Moreover they proposed that such core/mantle grains are the most reasonable candidates to reproduce the feature seen around $11.3 \mu \mathrm{m}$. They also suggested that radiative transfer calculations should be used in order to verify this model.

The main purpose of the present work is to present dust envelope models considering core/mantle grains composed by an $\alpha$-SiC core and an A.C. mantle. We also compare the data with models consisting of $\alpha$-SiC and A.C. homogeneous grains. Two samples of stars have been considered. The first one contains objects classified by SLMP. They were analyzed aiming to verify the classification proposed by the authors. The second sample contains 11 extreme carbon stars, which have thicker dust envelopes and a higher mass loss rate. The utilization of core/mantle grains is satisfactory to describe some stars but most of them are better reproduced using a two homogeneous grains model.

\section{Core/mantle grains and the method}

Besides carbon grains of different structures (e.g., graphite, amorphous carbon), $\mathrm{SiC}$ is the most important species for late-type carbon stars. $\mathrm{SiC}$ is one of the most refractory materials that may condense under the conditions of a carbon-rich chemistry. In such stars, almost all oxygen is chemically blocked in the CO molecule and, among the more abundant chemically active elements, carbon and $\mathrm{SiC}$ are the possible condensates. An important problem in the formation of $\mathrm{SiC}$ under the conditions present in circumstellar shells is that the abundance of $\mathrm{SiC}$ molecule is quite low. Beck (1992) has considered non-equilibrium effects and has shown that solid $\mathrm{SiC}$ can be stable against evaporation at temperatures below $1400 \mathrm{~K}$. He also suggested that $\mathrm{SiC}$ may form on the surface of preexisting carbon grains instead of being the primary condensate at very high temperatures. On the other hand, McCabe (1982) has shown that $\mathrm{SiC}$ particles can be formed at high temperatures due to the greenhouse effect. Following this suggestion, Kozasa et al. (1996) have demonstrated that the nucleation of $\mathrm{SiC}$ grains always precedes that of carbon grains when the non-LTE effect, i.e., the difference between the temperature of gas and small clusters, is taken into account.

We have considered this latest suggestion and modeled 45 envelopes of carbon stars using core/mantle grains consisting of a $\alpha$-SiC core and a A.C. mantle. The method employed is an improved version of that described by LorenzMartins \& Araújo (1997). We have modified the code to include a new option about grain properties. The absorption and scattering efficiencies, as well as the albedo, were calculated using the Mie theory for core/mantle grains (e.g. Bohren \& Huffman 1984; Hoyle \& Wickramasinghe 1991) and optical constants (or dielectric functions) tabulated in the literature. The optical constants which we have used are the ones determined by Pegourié (1988) for $\alpha$-SiC, and by Rouleau \& Martin (1991) for amorphous carbon.

The propagation of stellar and grain radiative energy is simulated photon by photon following a Monte Carlo scheme. For each interaction between a "photon" and a grain, a fraction of the energy is stored (absorption) and the remaining part is scattered according to the scattering diagram. The stellar radiation leads to an initial distribution of dust temperature and the thermal radiation from grains is simulated, giving after several iterations the equilibrium temperature. Computations give the spectral repartition of the total flux and of its different components (direct, scattered, emitted), and the temperature law for the grains. For more details see Lorenz-Martins \& Lefèvre (1993, 1994) and Lorenz-Martins \& Araújo (1997).

\section{The sample}

SLMP have studied 89 carbon-rich stars and organized the dust emission in several classes. Red class contains only 3 stars which present a $11.3 \mu \mathrm{m}$ feature and a strong dust continuum. SiC class is the most numerous, with 40 objects. They have the $11.3 \mu \mathrm{m}$ feature and a weak dust continuum. In the $\mathrm{SiC}+$ they put 32 stars which show two features: the $11.3 \mu \mathrm{m}$ one and a weak feature 
Table 1. Sample of SLMP's stars

\begin{tabular}{|c|c|c|c|c|c|c|}
\hline IRAS & Name & Sp.Type & Var. & Period & Env.class $^{i}$ & Phot. \\
\hline $00172+4425$ & VX And & $\mathrm{C} 4,5 \mathrm{~J}$ & SRa & $369^{a}$ & $\mathrm{SiC}++$ & 9 \\
\hline $01246-3248$ & R Scl & C6,5 & $\mathrm{SRb}$ & $370^{a}$ & $\mathrm{SiC}++$ & 1,7 \\
\hline $02270-2619$ & R For & $\mathrm{C} 4,3 \mathrm{e}$ & Mira & $388^{c}$ & $\mathrm{SiC}$ & 8 \\
\hline $03075+5742$ & $\mathrm{C}^{*} 131$ & $\mathrm{C} 4,5 \mathrm{~J}$ & $\mathrm{Lb}$ & - & $\mathrm{N}:$ & 9 \\
\hline $03374+6229$ & U Cam & $\mathrm{C} 6,4$ & $\mathrm{SRb}$ & - & $\mathrm{SiC}+$ & 9 \\
\hline $04459+6804$ & ST Cam & $\mathrm{C} 5,4$ & $\mathrm{SRb}$ & $300^{a}$ & $\mathrm{SiC}+:$ & 9 \\
\hline $04483+2826$ & TT Tau & $\mathrm{C} 7,4$ & $\mathrm{SRb}$ & $166^{a}$ & $\mathrm{SiC}+:$ & 9 \\
\hline $04573-1452$ & R Lep & $\mathrm{C} 7,4 \mathrm{e}$ & Mira & $427^{a}$ & $\mathrm{SiC}$ & 2,8 \\
\hline $05028+0106$ & W Ori & C5,4 & $\mathrm{SRb}$ & $212^{a}$ & $\mathrm{SiC}+$ & 2,9 \\
\hline $05418-4628$ & W Pic & $\mathrm{C}$ & $\mathrm{Lb}$ & - & $\mathrm{SiC}++$ : & 7 \\
\hline $05426+2040$ & Y Tau & $\mathrm{C} 6,4$ & $\mathrm{SRb}$ & $241^{a}$ & $\mathrm{SiC}$ & 7 \\
\hline $05576+3940$ & AZ Aur & $\mathrm{C} 8$ & Mira & $416^{a}$ & $\mathrm{Br} 2$ & 9 \\
\hline $06225+1445$ & BL Ori & $\mathrm{C} 6,3$ & $\mathrm{Lb}$ & - & $\mathrm{Br} 2$ & 3,9 \\
\hline $06331+3829$ & UU Aur & $\mathrm{C} 7,4$ & $\mathrm{SRb}$ & $23 a$ & $\mathrm{SiC}$ & 2,9 \\
\hline $06529+0626$ & CL Mon & $\mathrm{C} 6,3 \mathrm{e}$ & Mira & $497^{a}$ & $\mathrm{SiC}$ & 9 \\
\hline $07045-0728$ & RY Mon & C5,5 & $\mathrm{SRa}$ & $278^{a}$ & $\mathrm{SiC}+$ & 9,12 \\
\hline $07057-1150$ & W CMa & C6,3 & $\mathrm{Lb}$ & - & $\mathrm{SiC}+:$ & 7,9 \\
\hline $07065-7256$ & R Vol & $\mathrm{Ce}$ & Mira & $454^{a}$ & $\mathrm{SiC}$ & 5 \\
\hline $08538+2002$ & $\mathrm{~T}$ Cnc & $\mathrm{C} 5,5$ & $\mathrm{SRb}$ & $482^{a}$ & $\mathrm{SiC}++$ & 2,3 \\
\hline $09452+1330$ & IRC+10216 & $\mathrm{C} 9,4$ & Mira & $649^{c}$ & Red & 11 \\
\hline $10329-3918$ & U Ant & $\mathrm{C} 5,3$ & $\mathrm{Lb}$ & - & $\mathrm{SiC}+:$ & 7 \\
\hline $10350-1307$ & U Hya & $\mathrm{C} 5,3$ & $\mathrm{Lb}$ & - & $\mathrm{SiC}$ & $2,5,9$ \\
\hline $10491-2059$ & V Hya & $\mathrm{C} 6,5$ & SRa & $531^{a}$ & Red & $2,5,9$ \\
\hline $12226+0102$ & SS Vir & $\mathrm{C} 6,3$ & SRa & $364^{a}$ & Br1 & 9 \\
\hline $12427+4542$ & Y CVn & $\mathrm{C} 5,5 \mathrm{~J}$ & $\mathrm{SRb}$ & $158^{a}$ & $\mathrm{SiC}+:$ & $2,9,11$ \\
\hline $12447+0425$ & RU Vir & $\mathrm{C} 8,1 \mathrm{e}$ & Mira & $433^{a}$ & $\mathrm{SiC}$ & 5 \\
\hline $12544+6615$ & RY Dra & $\mathrm{C} 4,5 \mathrm{~J}$ & $\mathrm{SRb}$ & $200^{a}$ & $\mathrm{SiC}+$ & 2,9 \\
\hline $15094-6953$ & $\mathrm{X} \operatorname{Tr} \mathrm{A}$ & C5, 5 & $\mathrm{Lb}$ & - & $\mathrm{SiC}+$ & $5,11,12$ \\
\hline $18306+3657$ & T Lyr & $\mathrm{C} 6,5 \mathrm{~J}$ & $\mathrm{Lb}$ & - & $\mathrm{SiC}++$ & 9 \\
\hline $19017-0545$ & V Aql & C6,4 & $\mathrm{SRb}$ & $353^{a}$ & $\mathrm{SiC}+$ & 5,9 \\
\hline $19555+4407$ & AX Cyg & $\mathrm{C} 4,5$ & $\mathrm{Lb}$ & - & $\mathrm{SiC}+:$ & 9 \\
\hline $21032-0024$ & RV Aqr & $\mathrm{C} 6,3 \mathrm{e}$ & Mira & $454^{a}$ & $\mathrm{SiC}$ & 5 \\
\hline $21399+3516$ & V460Cyg & C6,4 & $\mathrm{SRb}$ & $263^{a}$ & $\mathrm{SiC}+:$ & 2,9 \\
\hline $23587+6004$ & WZ Cas & $\mathrm{C} 9,2 \mathrm{~J}$ & $\mathrm{SRb}$ & $186^{a}$ & Br1 & 3,10 \\
\hline
\end{tabular}

(1) Bagnulo et al. (1998); (2) Bergeat et al. (1976); (3) Bergeat \& Lunel (1980); (4) Epchtein et al. (1990); (5) Fouqué et al. (1992); (6) Jones et al. (1990); (7) Kerschbaum et al. (1996); (8) Le Bertre (1992); (9) Noguchi et al. (1981); (10) Noguchi \& Akiba (1986); (11) Nyman et al. (1992); (12) Walker (1976). Periodos: (a) Kholopov et al. (1987), (b) Jones et al. (1990), (c) Le Bertre (1992), (i) SLMP.

at 8-9 $\mu \mathrm{m}$. These objects also have a weak dust continuum. The $\mathrm{SiC}++$ class contains 6 stars with comparable $11.3 \mu \mathrm{m}$ and $8-9 \mu \mathrm{m}$ features. The five stars in the Broad 1 class have an unusual $11.3 \mu \mathrm{m}$ feature profile with shortwavelength excess. Finally, the Broad 2 class contains only 3 stars which present an unusual $11.3 \mu \mathrm{m}$ feature profile with long-wavelength excess.

In Table 1 we present our sample of 34 stars taken from SLMP. We restrict our study to the objects with IR fluxes published in the literature. Fortunately, we have been able to obtain data from stars belonging to all different classes. Table 1 lists the IRAS number (Col. 1) followed by the usual name (Col. 2). Spectral type and variability class are listed in Cols. 3 and 4 respectively. Column 5 gives the period and Col. 6 shows the envelope class attributed by SLMP. Some stars have a doubtful classification; they are designated by a colon. Finally the photometry used in the fit of the models is presented in the last column. Table 2 list the extreme carbon stars sample. The columns are analogous to those of Table 1 . The stars analyzed are variables and self-consistent models for them require data from similar phase of luminosity. However this is difficult due to the scarcity of observations. We have worked with the photometry available in the literature and considered the phase whenever possible. In order to minimize the uncertainties, we have used average LRS (IRAS, 1986) spectra for $\mathrm{SiC}$ emission. The 12, 25, 60, $100 \mu \mathrm{m}$ fluxes were taken from Gezari et al. (1987) and SIMBAD.

\section{Results}

Tables 3 and 4 present the results of the best models to our first and second samples, respectively. These results were 
Table 2. Sample with evolved stars

\begin{tabular}{lllll}
\hline IRAS & Name & Sp.Type & Period & Phot. \\
\hline $05377+1346$ & AFGL 799 & C8,4 & $372^{c}$ & 1,4 \\
$05405+3240$ & AFGL 809 & C & $780^{b}$ & 5 \\
$06012+0726$ & AFGL 865 & $?$ & $696^{c}$ & 6 \\
$06291+4319$ & AFGL 954 & C & - & 5 \\
$06342+0328$ & AFGL 971 & C & $653^{c}$ & 6 \\
$07098-2112$ & AFGL 1085 & N & $725^{c}$ & 6 \\
$08088-3243$ & AFGL 1235 & C & $571^{c}$ & 2 \\
$15082-4808$ & AFGL 4211 & $?$ & - & 3 \\
$19594+4047$ & AFGL 2494 & C & $783^{b}$ & 5 \\
$20570+2714$ & AFGL 2686 & C8,5 & $750^{b}$ & 5 \\
$23257+1038$ & AFGL 3099 & C & $484^{c}$ & 5 \\
\hline
\end{tabular}

(1) Cohen (1984); (2) Epchtein et al. (1990); (3) Fouqué et al. (1992); (4) Gehrz \& Hackwell (1976); (5) Jones et al. (1990); (6) Le Bertre (1992); Periods: (a) Kholopov et al. (1987), (b) Jones et al. (1990), (c) Le Bertre (1992).

obtained considering a core/mantle grain. IRAS number (Col. 1) is followed by the temperature of the central star $\left(T_{\text {eff }}\right.$ in $\left.\mathrm{K}\right)$ in the second column. Third and fourth columns present inner $\left(R_{1}\right.$ in $\left.R_{\star}\right)$ and outer $\left(R_{2}\right.$ in $\left.R_{\star}\right)$ envelope radii, respectively. The dimension of the $\alpha$-SiC core $\left(c_{\mathrm{SiC}}\right.$ in $\left.\AA\right)$ of the grains is given in the fifth column, followed by the dimension of the amorphous carbon mantle $\left(m_{\text {A.C. }}\right.$ in $\left.\AA\right)$ in the sixth column. Finally, the optical depth $(\tau)$ at $1 \mu \mathrm{m}$ and the abundance ratio between $\alpha$ $\mathrm{SiC}$ and amorphous carbon grains are given, respectively, in Cols. 7 and 8. In Table 3 we have added one last column with the SLMP's envelope classes.

In addition, we have calculated models for the stars in the sample using a code with two homogeneous grains. Some stars of this sample were analyzed in Lorenz-Martins \& Lefèvre (1994), where the authors describe the method. The differences between parameters of both codes are the size of the grains and the way in which the $\mathrm{SiC} / \mathrm{A}$.C. ratios were calculated. In the core/mantle models, we consider the core $\left(c_{\mathrm{SiC}}\right)$ and mantle $\left(m_{\mathrm{A} . \mathrm{C} .}\right)$ size. $\mathrm{SiC} /$ A.C. ratios in this method are obtained by mass, based on the mantle and core size, and we find the corresponding value as obtained in the two homogeneous grains model. Others parameters are obtained the same way as in the two homogeneous grains code.

The features seen in the mid-IR LRS must be reproduced and the properties of complete CDS must be determined simultaneously. In order to fit the dust emission, we have calculated grids of about fifty models for each star, and we inspect visually the model which best reproduces the complete CDS. We pay special attention to the LRS feature and accept errors of about 15 per cent in all parameters (such as grain size, effective temperature, optical depth...) to fit this emission feature; in fact it is this feature that defines the best model.
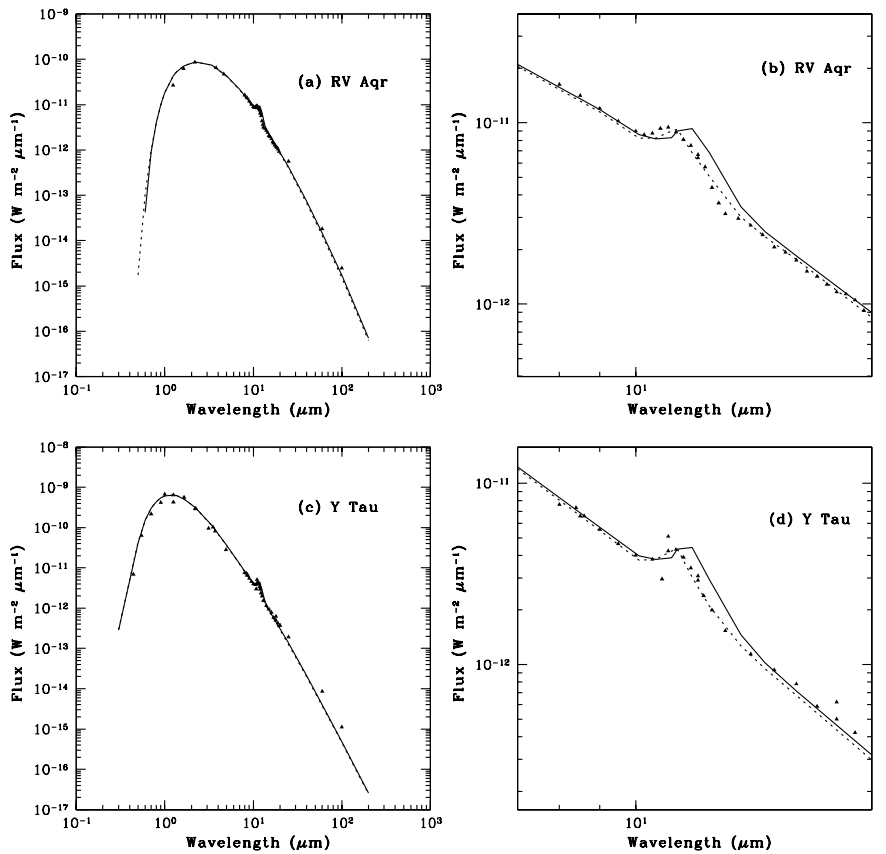

Fig. 1. Best models for SiC class. In Figs. 1a-d we plotted the best models using core/mantle grains (solid line) and two homogeneous grain model (dashed line). Triangles represent the photometric data. In Figs. 1b and 1d we show an enlarged view of the $11.3 \mu \mathrm{m}$ emission

\subsection{Results for SLMP stars}

\section{$\mathrm{SiC}$ Class}

We analyzed 9 out of 40 SLMP objects in this class. We found that effective temperatures vary between $2200 \mathrm{~K}$ and $2700 \mathrm{~K}$. Inner radii vary between $3.7 R_{*}$ and $5.0 R_{*}$, while most of the outer radii are $1000 R_{*}$. It must be kept in mind that the results are not very sensitive to this last parameter, as has been pointed out in previous works. The $\alpha$-SiC core $\left(c_{\mathrm{SiC}}\right)$ grains vary between 280 to $550 \AA$, and the amorphous carbon mantle $\left(m_{\text {A.C. }}\right)$ between 700 and $1050 \AA$. Optical depths for this class vary between 0.10 and 2.5, with two exceptions: U Hya $(\tau=0.03)$ and $R$ for $(\tau=7.0)$. Finally SiC/A.C. ratios vary from 0.06 to 0.20 . In Figs. 1a-d we show out fits to RV Aqr and Y Tau. Solid lines represent the core/mantle grain model and dashed lines the two homogeneous grains model. Figures $1 \mathrm{~b}$ and $1 \mathrm{~d}$ show an enlarged view centered on the $11.3 \mu \mathrm{m}$ feature. In all cases, best fits to this class were obtained using the two homogeneous grains model.

\section{$\mathrm{SiC}+$ Class}

We have modeled 7 out 32 SLMP SiC+ stars. According to our results, the temperatures of central stars vary from $2400 \mathrm{~K}$ to $2650 \mathrm{~K}$. Outer dust envelope radii are the same for all stars $\left(R_{2}=1000 R_{*}\right)$ and inner radii vary between $3.7 R_{*}$ and $6.5 R_{*}$. Sizes of mantle grains $\left(m_{\text {A.C. }}\right)$ show a great dispersion: $400 \AA$ to $1200 \AA$. The same occurs with the sizes of $\alpha$-SiC core $\left(c_{\mathrm{SiC}}\right)$ which vary from $155 \AA$ to $500 \AA$. Optical depths have values between 0.03 and 0.50 , and $\mathrm{SiC} / \mathrm{A}$.C. ratios between 0.06 and 0.26. Almost all stars were best described using the two homogeneous 
Table 3. Results of first sample

\begin{tabular}{|c|c|c|c|c|c|c|c|c|}
\hline Stars & $T_{\text {eff }}(\mathrm{K})$ & $R_{1}\left(R_{\star}\right)$ & $R_{2}\left(R_{\star}\right)$ & $c_{\mathrm{SiC}}(\AA)$ & $m_{\text {A.C. }}(\AA)$ & $\tau$ & SiC/A.C. & Env. Class. \\
\hline $00172+4425$ & 2200 & 5.0 & 1000 & 453 & 1000 & 0.02 & 0.10 & $\mathrm{SiC}++$ \\
\hline $01246-3248$ & 2400 & 5.0 & 1000 & 500 & 1200 & 0.10 & 0.08 & $\mathrm{SiC}++$ \\
\hline $02270-2619$ & 2500 & 5.0 & 5000 & 407 & 1050 & 7.00 & 0.06 & $\mathrm{SiC}$ \\
\hline $03075+5742$ & 2400 & 5.0 & 800 & 453 & 1000 & 0.01 & 0.10 & $\mathrm{~N}:$ \\
\hline $03374+6229$ & 2650 & 4.6 & 1000 & 500 & 1200 & 0.50 & 0.08 & $\mathrm{SiC}+$ \\
\hline $04459+6804$ & 2700 & 4.8 & 1000 & 500 & 850 & 0.03 & 0.26 & $\mathrm{SiC}+:$ \\
\hline $04483+2826$ & 2650 & 3.0 & 1000 & 236 & 400 & 0.02 & 0.26 & $\mathrm{SiC}+:$ \\
\hline $04573-1452$ & 2250 & 5.0 & 1000 & 294 & 700 & 0.60 & 0.08 & $\mathrm{SiC}$ \\
\hline $05028+0106$ & 2650 & 5.2 & 1000 & 332 & 700 & 0.10 & 0.12 & $\mathrm{SiC}+$ \\
\hline $05418-4628$ & 2400 & 5.0 & 1000 & 500 & 1200 & 0.10 & 0.08 & $\mathrm{SiC}++$ : \\
\hline $05426+2040$ & 2600 & 4.5 & 1000 & 554 & 1000 & 0.20 & 0.20 & $\mathrm{SiC}$ \\
\hline $05576+3940$ & 2200 & 4.6 & 1000 & 403 & 1000 & 1.20 & 0.07 & Br2 \\
\hline $06225+1445$ & 2700 & 4.9 & 1000 & 400 & 850 & 0.03 & 0.12 & $\mathrm{Br} 2$ \\
\hline $06331+3829$ & 2550 & 3.7 & 1000 & 340 & 700 & 0.10 & 0.13 & $\mathrm{SiC}$ \\
\hline $06529+0626$ & 2200 & 4.6 & 1000 & 282 & 700 & 1.00 & 0.07 & $\mathrm{SiC}$ \\
\hline $07045-0728$ & 2400 & 6.5 & 1000 & 270 & 500 & 0.10 & 0.18 & $\mathrm{SiC}+$ \\
\hline $07057-1150$ & 2650 & 3.0 & 1000 & 440 & 740 & 0.04 & 0.27 & $\mathrm{SiC}+:$ \\
\hline $07065-7256$ & 2400 & 4.6 & 1000 & 282 & 700 & 2.20 & 0.07 & $\mathrm{SiC}$ \\
\hline $08538+2002$ & 2400 & 5.0 & 1000 & 400 & 1200 & 0.20 & 0.04 & $\mathrm{SiC}++$ \\
\hline $09452+1330$ & 2100 & 5.5 & 8000 & 110 & 500 & 10.0 & 0.01 & Red \\
\hline $10329-3918$ & 2700 & 4.5 & 1000 & 390 & 850 & 0.04 & 0.11 & $\mathrm{SiC}+:$ \\
\hline $10350-1307$ & 2700 & 4.5 & 1000 & 468 & 850 & 0.03 & 0.20 & $\mathrm{SiC}$ \\
\hline $10491-2059$ & 2050 & 5.6 & 10000 & 270 & 950 & 0.70 & 0.02 & Red \\
\hline $12226+0102$ & 2700 & 5.0 & 1000 & 487 & 1200 & 0.35 & 0.07 & Br1 \\
\hline $12427+4542$ & 2700 & 4.9 & 1000 & 317 & 700 & 0.05 & 0.10 & $\mathrm{SiC}+:$ \\
\hline $12447+0425$ & 2200 & 4.3 & 1000 & 307 & 700 & 2.50 & 0.09 & $\mathrm{SiC}$ \\
\hline $12544+6615$ & 2650 & 3.7 & 1000 & 155 & 400 & 0.04 & 0.06 & $\mathrm{SiC}+$ \\
\hline $15094-6953$ & 2650 & 5.3 & 1000 & 416 & 700 & 0.03 & 0.26 & $\mathrm{SiC}+$ \\
\hline $18306+3657$ & 2200 & 5.0 & 1000 & 317 & 700 & 0.03 & 0.10 & $\mathrm{SiC}++$ \\
\hline $19017-0545$ & 2550 & 4.9 & 1000 & 315 & 800 & 0.10 & 0.07 & $\mathrm{SiC}+$ \\
\hline $19555+4407$ & 2400 & 4.0 & 1000 & 761 & 1500 & 0.08 & 0.15 & $\mathrm{SiC}+:$ \\
\hline $21032-0024$ & 2200 & 4.5 & 1000 & 294 & 700 & 2.50 & 0.08 & $\mathrm{SiC}$ \\
\hline $21399+3516$ & 2800 & 4.0 & 10000 & 500 & 1000 & 0.04 & 0.14 & $\mathrm{SiC}+:$ \\
\hline $23587+6004$ & 2500 & 3.0 & 800 & 554 & 1000 & 0.01 & 0.20 & Br1 \\
\hline
\end{tabular}

grains model; the unique exception is $\mathrm{V}$ Aql, which is better reproduced with a core/mantle grain code. Figure 2 shows best fits to RY Mon and U Cam.

As a rule, we can say that the envelope of $\mathrm{SiC}$ and $\mathrm{SiC}+$ stars are nicely described by the existence of $\alpha-\mathrm{SiC}$ and A.C. homogeneous grains simultaneously.

\section{$\mathrm{SiC}++$ Class}

We analyzed 5 out of 6 SLMP objects. According to our models, effective temperatures are either $2200 \mathrm{~K}$ or $2400 \mathrm{~K}$. Almost all inner and outer radii have the same values: $R_{1}=5 R_{*}$ and $R_{2}=1000 R_{*}$. Mantle sizes $\left(m_{\mathrm{A} . \mathrm{C} .}\right)$ vary between 700 and $1200 \AA$, and core sizes $\left(c_{\mathrm{SiC}}\right)$ between 300 and $500 \AA$. Optical depths vary between 0.02 and 0.20 , which indicates very thin dust envelopes, and $\mathrm{SiC} / \mathrm{A}$.C. ratios between 0.04 and 0.10 . Due to the absence of optical constants to describe the $8-9 \mu \mathrm{m}$ and $13 \mu \mathrm{m}$ features, it is difficult to choose between the core/mantle grain or the two homogeneous grains model. We will discuss these results in the next section. Figure 3 shows two stars of this class.

\section{Broad 1 and Broad 2 Classes}

We have modeled 2 out 5 SLMP Broad 1 , and 2 out 3 SLMP Broad 2 stars. Our results indicate very similar properties for both classes. The temperatures of the central stars are in the range of $2200 \mathrm{~K}$ to $2700 \mathrm{~K}$. Core and mantle sizes vary between $400-500 \AA$ and $850-1200 \AA$, respectively. They have thin envelopes with analogous dimensions. SiC/A.C. ratios varying between 0.07 and 0.20 were found. We have obtained our best fits using a two homogeneous grains code for 3 stars. WZ Cas presents an absorption at about $14 \mu \mathrm{m}$, which is usually seen in J-type carbon stars, and it's difficult to distinguish between the models. SS Vir shows features at about $8 \mu \mathrm{m}$ and $14 \mu \mathrm{m}$, too. Figure 4 presents best fit models to SS Vir (Broad 1 class) and AZ Aur (Broad 2 class).

\section{Red Class}

We have modeled 2 out of 3 SLMP stars: IRC+10216 and $\mathrm{V}$ Hya. Both objects are very well studied and supposed to have asymmetrical dust envelopes. They are believed to be in the latest stages of stellar carbon evolution. We will discuss them below. 

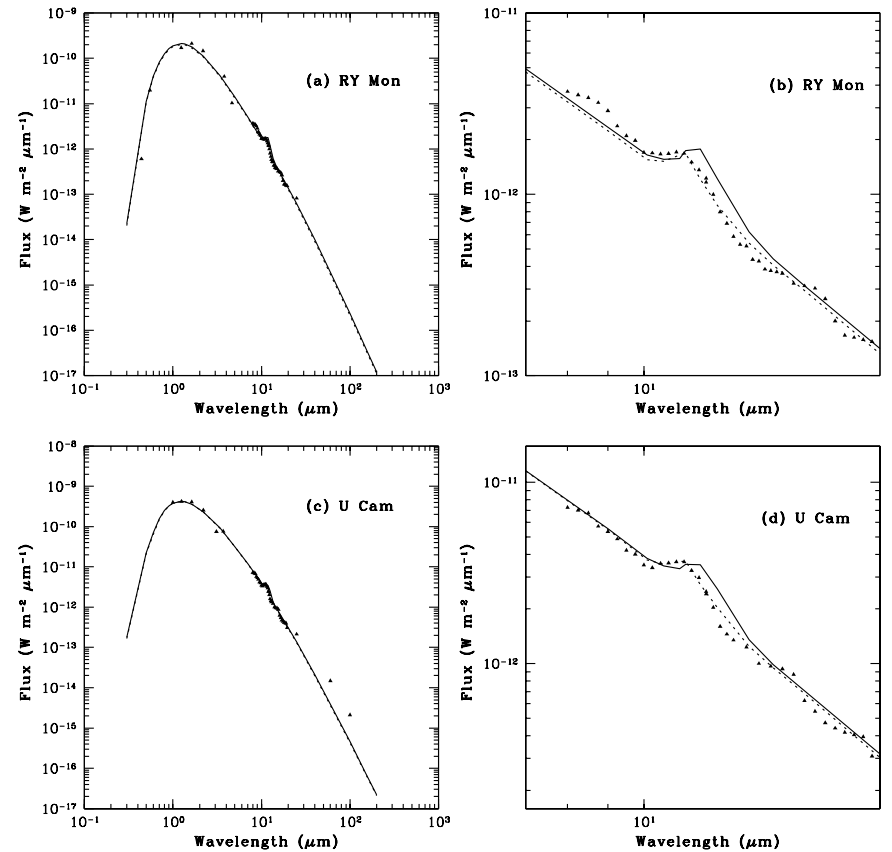

Fig. 2. This figure shows the best models for RY Mon and $\mathrm{U}$ Cam, which are in the $\mathrm{SiC}+$ class. In Figs. $2 \mathrm{a}-\mathrm{d}$ we plotted models using core/mantle grain (solid line) and two homogeneous grain model (dashed line). Triangles represent the photometric data. In Figs. $2 \mathrm{~b}$ and $2 \mathrm{~d}$ we show an enlarged view of the $11.3 \mu \mathrm{m}$ emission

\section{(a) IRC+10216}

IRC +10216 has been extensively observed at optical, radio and infrared wavelengths. The central object is a long-period variable, with a period of 640 days, and it is commonly considered to be a late-type carbon star. Its envelope has been continuously surveyed and 380 molecular lines were detected, of which 317 have been identified (Cernicharo et al. 2000). Deep $B$ and $V$ image-bands, reveal its extended circumstellar envelope in the dust scattered and show an episodic mass loss rate. The circumstellar envelope is roughly spherically symmetrical but it is likely to be composed of a series of discrete, incomplete, concentric shells (Mauron \& Huggins 1999).

IRC +10216 was modeled by several authors. Michell \& Robinson (1980) have used graphite grains and radiative transfer calculations were performed considering, as usual, a spherically symmetric envelope with a central star. Rowan-Robinson \& Harris (1983), Le Bertre (1987, 1988b), Martin \& Rogers (1987) and Griffin (1990) treated radiative transfer in the envelope of this star in a similar way. Lorenz-Martins \& Lefèvre (1993, 1994) have modeled this star by considering a two homogeneous grain model consisting of $\alpha$-SiC and amorphous carbon grains simultaneously, as already cited. More recently, Groenewegen (1997) has computed a spherically symmetrical dust model and suggested that $\mathrm{IRC}+10216$ is in the latest phases of carbon stars evolution, like $\mathrm{V}$ Hya.

The effective temperature of IRC+10216 is $2100 \mathrm{~K}$ and our results lead to an extensive $\left(R_{1}=5.6 R_{*}\right.$ and $R_{2}=$ $\left.8000 R_{*}\right)$ but thick $(\tau=10)$ dust envelope. The size of
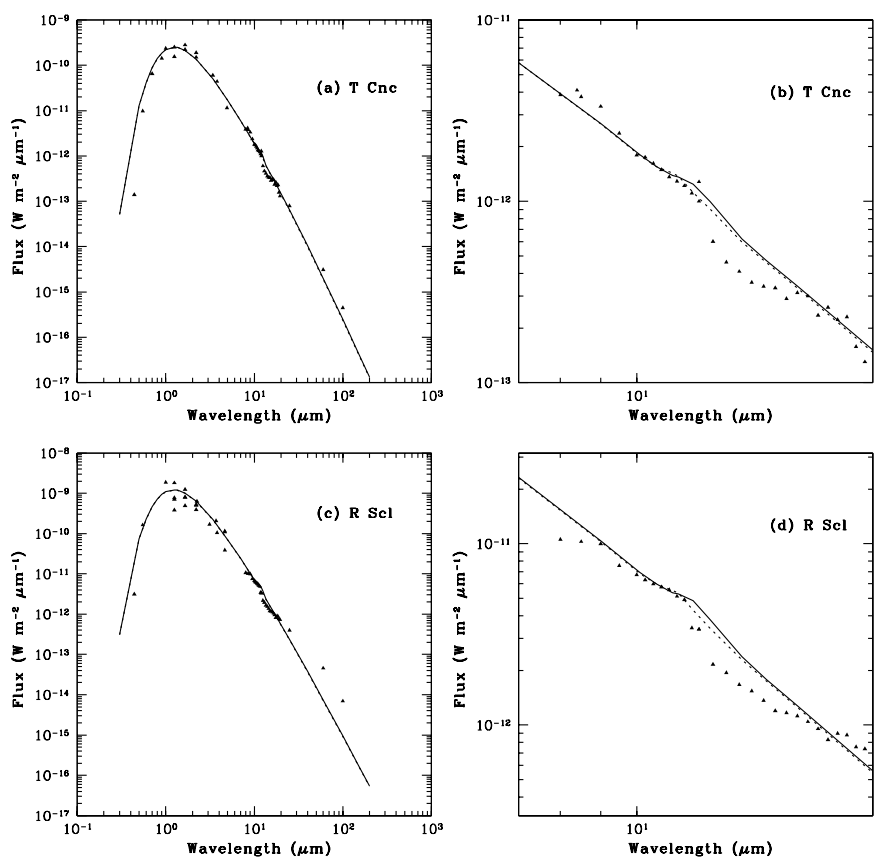

Fig. 3. This figure shows best models for TCnc and R Scl, which are in $\mathrm{SiC}++$ class. In Figs. 3a-d we plotted models using core/mantle grain (solid line) and two homogeneous grain model (dashed line). Triangles represent the photometric data. In Figs. $3 \mathrm{~b}$ and $3 \mathrm{~d}$ we show an enlarged view of the $11.3 \mu \mathrm{m}$ emission
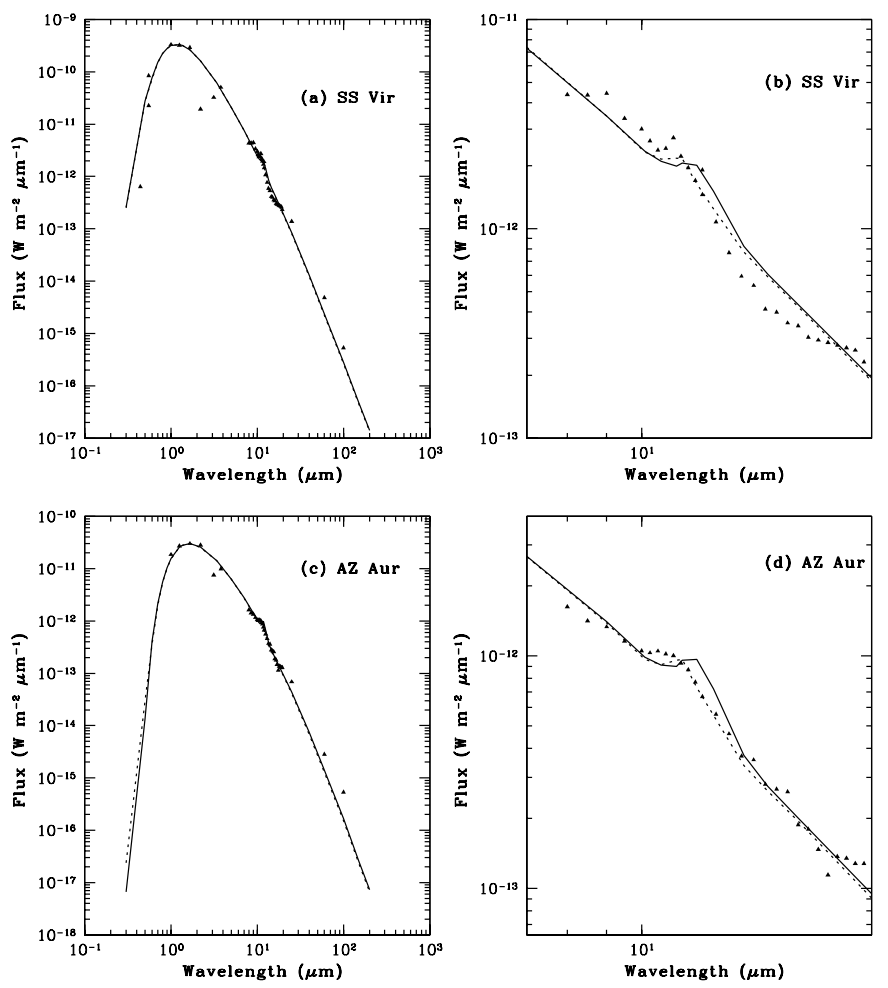

Fig. 4. This figure shows best models for SS Vir, which belongs to Broad 1 class and AZ Aur to Broad 2 class. In Figs. 4a-d we plotted models using core/mantle grain (solid line) and two homogeneous grain model (dashed line). Triangles represent the photometric data. In Figs. $4 \mathrm{~b}$ and $4 \mathrm{~d}$ we show an enlarged view of the $11.3 \mu \mathrm{m}$ emission 
the amorphous carbon mantle grains $\left(m_{\text {A.C. }}\right)$ was $500 \AA$ with an $\alpha$-SiC core of $110 \AA$. Our best model was obtained considering core/mantle grains as can be seen in Figs. 5a and $5 \mathrm{~b}$.

\section{(b) V Hya}

V Hya was classified as C6,5 by Yamashita (1972). It is a variable star with overlapping periods: a period of about 530 days with amplitude of about $1.5 \mathrm{mg}$ and a longer period of 6500 days with amplitude of $3.5 \mathrm{mg}$. This object is surrounded by an extended expanding molecular envelope, resulting from extensive mass-loss. Massloss rate is in the range of 3.0-4.0 $10^{-6} M_{\odot} / \mathrm{yr}$ (Knapp \& Morris 1985; Olofsson et al. 1990). Polarimetric measures have been obtained by Johnson \& Jones (1991) and more recently by Trammell et al. (1994). Johnson \& Jones (1991) have classified V Hya as a proto-planetary nebula and measured $P(V)=0.75 \% \pm 0.02 \%$ at $\theta=21^{\circ} \pm 1^{\circ}$. Trammell et al. (1994) have observed this object in April 1992 and January 1993, and found that the polarization varied over this interval. The envelope properties found from molecular line observations by Knapp et al. (2000), like the fast molecular wind and the high mass loss rate, suggest that V Hya has entered its "superwind" phase. However, its spectral type, period, colors, and lack of ionizing radiation indicate that this star is still on the AGB. Then, V Hya is believed to be in the latest phases of mass loss on the AGB.

Our results show that the temperature of the central star is $2050 \mathrm{~K}$. Contrary to what is expected for this evolved phase, we have found a thin $(\tau=0.7)$ and extensive dust envelope $\left(R_{1}=5.5 R_{*}\right.$ and $\left.R_{2}=10000 R_{*}\right)$. The size of the amorphous carbon mantle $\left(m_{\text {A.C. }}\right)$ is $950 \AA$ and the $\alpha$ - $\mathrm{SiC}$ core is $270 \AA$. $\mathrm{SiC} / \mathrm{A}$.C. ratio is very small, 0.02 . The best fit can be seen in Figs. $5 \mathrm{c}$ and $5 \mathrm{~d}$. We can say that a core/mantle grain model fits this star well, but a two homogeneous grain model cannot be discarded.

\section{$\mathrm{SiC}+$ : Stars}

We analyzed 7 out 11 SLMP objects classified as $\mathrm{SiC}+$ :, where the colon means a more uncertain classification. Almost all stars are well reproduced by a core/mantle grain model. The only exception is AX Cyg, which seems to need single particles of $\alpha$-SiC, amorphous carbon and core/mantle grains simultaneously. The results obtained with the core/mantle grain code are similar to those found for $\mathrm{SiC}+$ class stars. The temperatures of the central stars vary from $2400 \mathrm{~K}$ to $2800 \mathrm{~K}$. Inner radii vary between 3 to $4.9 R_{\star}$. Carbon mantle sizes $\left(m_{\text {A.C. }}\right)$ vary from $400 \AA$ to $1500 \AA$ with core $\left(c_{\mathrm{SiC}}\right)$ values between $236 \AA$ and $761 \AA$. Optical depths are lower: $0.02 \leq \tau \leq 0.08$. On the other hand $\mathrm{SiC} /$ A.C. abundance ratios vary from 0.10 to 0.27 . (For these stars we could say that the core/mantle grain models are more adequate, even with such small optical depths, since the $\mathrm{SiC} /$ A.C. ratios are higher.) Figure 6 shows an enlarged view of the two stars in this class, Y CVn and W CMa. We can see that the emission feature is shifted to longer wavelengths. This is a result of
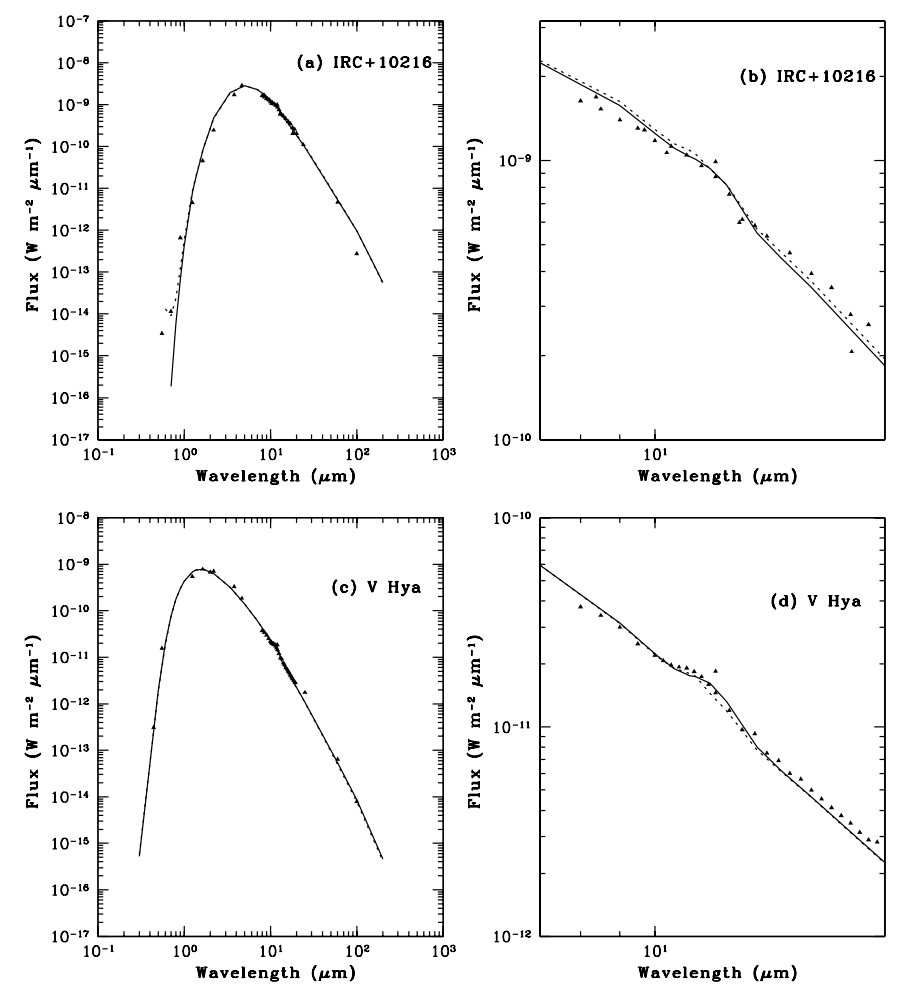

Fig. 5. This figure shows the best models for IRC+10216 and VHya which belong to Red class. In Figs. 5a-d we plotted models using core/mantle grain (solid line) and two homogeneous grain model (dashed line). Triangles represent the photometric data. In Figs. 5b and 5d we show an enlarged view of the $11.3 \mu \mathrm{m}$ emission

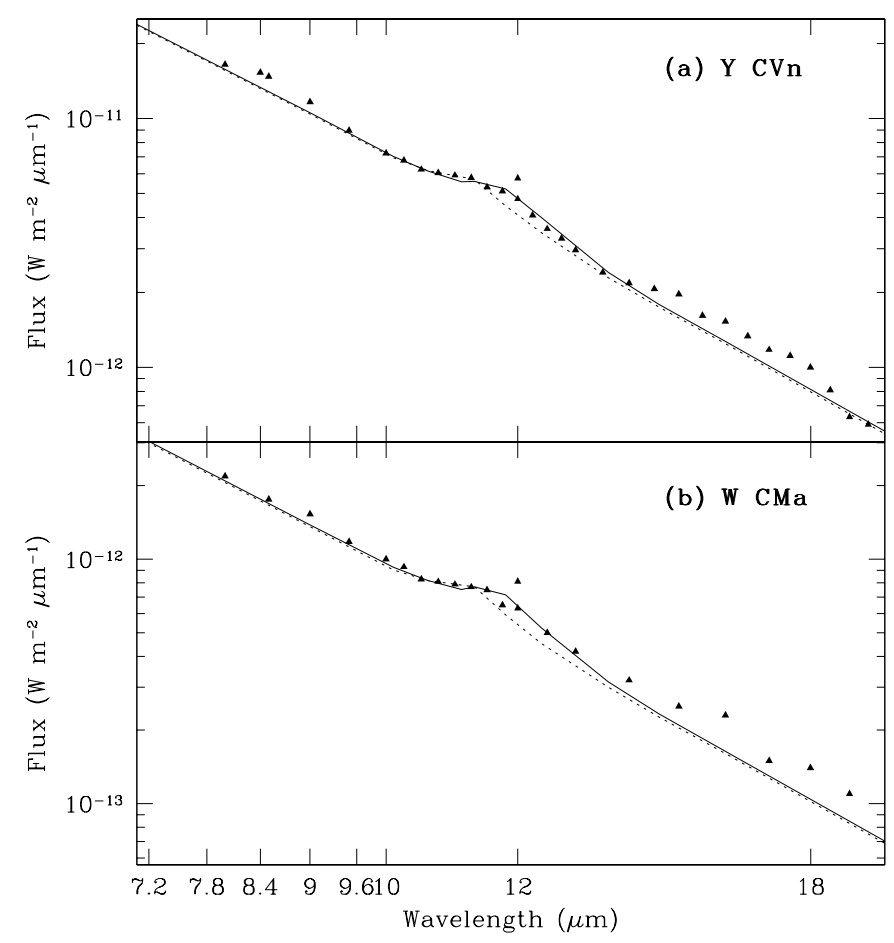

Fig. 6. This figure shows best models for Y Cvn and W CMa which belong to $\mathrm{SiC}+$ : class. In Figs. $6 \mathrm{a}-\mathrm{b}$ we plotted models using core/mantle grain (solid line) and two homogeneous grain model (dashed line). Triangles represent the photometric data 
Table 4. Results of the Sample with evolved stars

\begin{tabular}{lllllcrrr}
\hline IRAS & $T_{\text {eff }}(\mathrm{K})$ & $R_{1}\left(R_{\star}\right)$ & $R_{1}\left(\mathrm{R}_{\star}\right)$ & $c_{\mathrm{SiC}}(\AA)$ & $m_{\mathrm{A} . \mathrm{C} .}(\AA)$ & $\tau$ & $\mathrm{SiC} / \mathrm{AC}$ & Env. Class \\
\hline $05377+1346$ & 2350 & 3.0 & 2000 & 282 & 700 & 3.0 & 0.07 & $\mathrm{E} 1$ \\
$05405+3240$ & 2200 & 7.1 & 10000 & 215 & 700 & 10.6 & 0.03 & $\mathrm{E} 2$ \\
$06012+0726$ & 2000 & 4.5 & 4000 & 189 & 900 & 12.5 & 0.01 & $\mathrm{E} 2$ \\
$06291+4319$ & 2300 & 6.5 & 1000 & 226 & 700 & 3.5 & 0.04 & $\mathrm{E} 1$ \\
$06342+0328$ & 2500 & 4.0 & 7000 & 256 & 700 & 7.0 & 0.05 & $\mathrm{E} 2$ \\
$07098-2112$ & 2450 & 6.5 & 1000 & 236 & 700 & 4.0 & 0.04 & $\mathrm{E} 1$ \\
$08088-3243$ & 2300 & 6.4 & 1000 & 215 & 700 & 4.0 & 0.03 & $\mathrm{E} 1$ \\
$15082-4808$ & 2050 & 4.6 & 1000 & 273 & 1000 & 9.4 & 0.02 & $\mathrm{E} 2$ \\
$19594+4047$ & 2000 & 5.0 & 7000 & 232 & 800 & 13.0 & 0.025 & $\mathrm{E} 2$ \\
$20570+2714$ & 2200 & 8.0 & 1000 & 156 & 500 & 5.2 & 0.03 & $\mathrm{E} 1$ \\
$23257+1038$ & 1900 & 7.0 & 1000 & 254 & 700 & 13.0 & 0.05 & $\mathrm{E} 2$ \\
\hline
\end{tabular}

Mie's theory applied to core/mantle spheres. Suh (2000) has found a similar behavior in his models.

\subsection{Extreme carbon stars}

Except for one case (R For), all SiC classes of the SLMP sample contains carbon stars which have thin dust envelopes $(\tau \leq 2.5)$. Our sample of extreme carbon stars contains objects that have optical depths varying between 3.0 and 13. In order to make our analysis easier, we have decided to separate them according to this physical quantity: $3.0 \leq \tau \leq 5.2$ (E1 group) and $7.0 \leq \tau \leq 13.0$ (E2 group).

The stars belonging to our E1 group were better described by a two homogeneous grains model. Temperatures of the central stars are between $2200 \mathrm{~K}$ and $2450 \mathrm{~K}$. Inner radii are about $6.5 R_{*}$ and outer radii $1000 R_{*}$ for all stars. Core $\left(c_{\mathrm{SiC}}\right)$ and mantle $\left(m_{\mathrm{A} . \mathrm{C}}\right)$ sizes are respectively about $200 \AA$ and $700 \AA$ in almost all cases. The abundance ratios SiC/A.C. vary from 0.03 and 0.07 . These results can indicate that such stars are related to the SiC SLMP class.

On the contrary, stars belonging to our E2 group were better fitted with the core/mantle grain code and are similar to the stars in the Red class. They are cooler $(1900 \mathrm{~K} \leq$ $T_{\text {eff }} \leq 2200 \mathrm{~K}$ ) and present a dust envelope more extensive than those of the E1 group. Core and mantle sizes vary between 190 to $270 \AA$ and $700 \AA$ to $1000 \AA$, respectively. The $\mathrm{SiC} / \mathrm{A}$.C. abundance ratios are also low, with values between 0.01 and 0.05 . In the E2 group, three stars were better represented by taking into account $\alpha$-SiC, amorphous carbon and core/mantle grains simultaneously. We can speculate that these three stars represent a transition phase between $\mathrm{SiC}$ and Red classes. Figure 7 shows an enlarged view of AFGL 954 (E1 group) and AFGL 809 (E2 group).

\section{Discussion and conclusions}

As commented in the previous section, the IR fluxes and the feature around $11.3 \mu \mathrm{m}$ present in most sources that we have analyzed, are likely to be better reproduced using

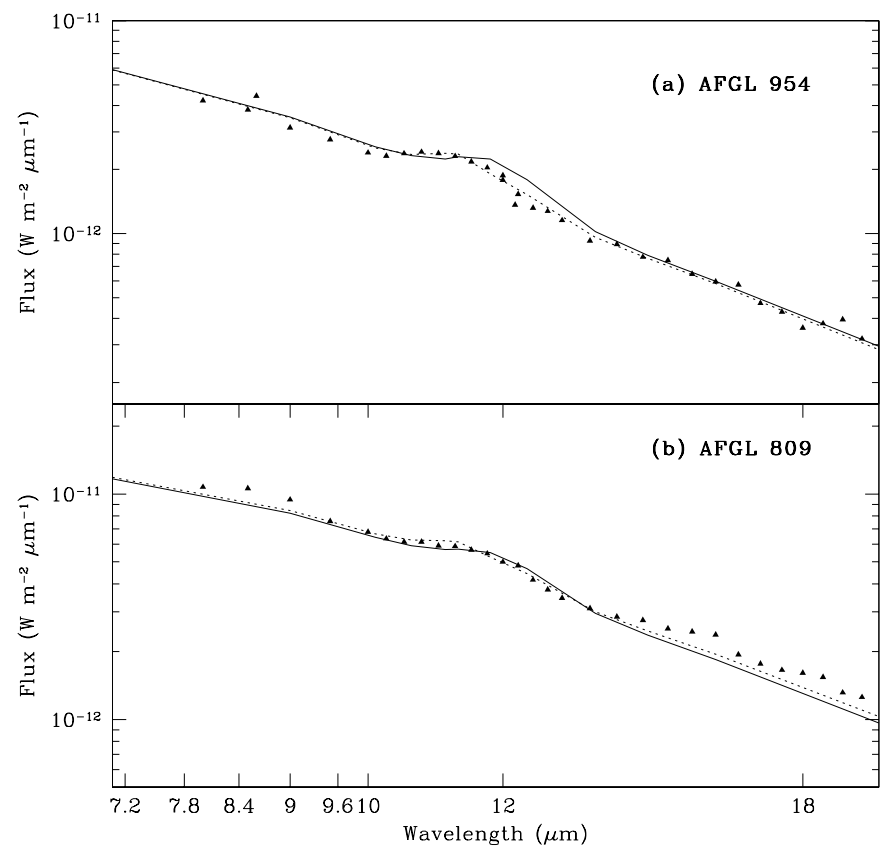

Fig. 7. This figure shows the best models for AFGL 954 and AFGL 809 which belong to E1 and E2 classes respectively. In Figs. 7a-b we plotted models using core/mantle grain (solid line) and two homogeneous grain model (dashed line). Triangles represent the photometric data

two homogeneous grains (A.C. and $\alpha$-SiC) simultaneously. This is true for $\mathrm{SiC}, \mathrm{SiC}+$ and our E1 stars. On the other hand, a few objects (those in Red and in our E2 class) are better described by a core/mantle grain model. However, the $\mathrm{SiC}++$ and Broad 1 classes cannot be reproduced with the existing optical constants.

The $\mathrm{SiC}++$ class presents both the $8-9 \mu \mathrm{m}$ emission and also a very prominent $14 \mu \mathrm{m}$ absorption feature. The stars belonging to the Broad 1 class also show a $14 \mu \mathrm{m}$ absorption. This absorption feature is weaker in Mira variables than in SR variables. This may be explained by a stronger dust emission in Mira variables which fills the molecular absorption (see Yamamura et al. 1998). Regarding the origin of the $8-9 \mu \mathrm{m}$ emission feature, Aoki et al. (1999) have suggested that it may be a 
result of molecular absorption at $7.5 \mu \mathrm{m}$ and $\mathrm{SiC}$ emission at $11.3 \mu \mathrm{m}$. This absorption could be due to $\mathrm{HCN}$ and/or $\mathrm{C}_{2} \mathrm{H}_{2}$ photospheric absorption bands. On the other hand, the absorption feature at about $14 \mu \mathrm{m}$ was attributed by the same authors to $\mathrm{HCN}$ and $\mathrm{C}_{2} \mathrm{H}_{2}$ absorption in the photosphere or in the warm envelope close to the star. This absorption feature would be formed in the inner envelope where the mid-infrared radiation originates. On the other hand, Yamamura et al. (1998) have studied the $14 \mu \mathrm{m}$ absorption in the ISO SWS spectra of 11 carbon stars with mass-loss ranging from $10^{-8}$ to $10^{-4} M_{\odot} /$ yr. According to these authors, all stars clearly show an absorption band at about $13.7 \mu \mathrm{m}$ due to $\mathrm{C}_{2} \mathrm{H}_{2}$ while the contribution from $\mathrm{HCN}$ molecules is small in this region.

The stars of the $\mathrm{SiC}++$ class have thin envelopes that can favor the misidentification of spectral features like the $8-9 \mu \mathrm{m}$ one. On the other hand, this feature is also observed in some dust-enshrouded carbon stars, such as IRAS 15194-5115, IRAS 18239-0655 and IRAS $18240+2326$. Consequently, this feature could perhaps be produced by a solid component. Indeed, Goebel et al. (1995) have suggested $\alpha: \mathrm{C}-\mathrm{H}$, as described by Dischler et al. (1983). Unfortunately, they have not published the set of optical constants to this component. In order to try to reproduce the $8-9 \mu \mathrm{m}$ emission, we have modeled SS Vir, considering optical constants of the amorphous HAC as tabulated by Zubko (1996), but we have not been able to fit the emission. Bagnulo et al. (1998) have shown that this feature can be reproduced by a dust shell composed of a mixture of $\mathrm{SiC}$ grains and silicate grains. This interpretation, however, does not seem to be consistent with the theory of dust formation. Before discussing such results, let us comment now on some alternatives that might be investigated.

On the basis of a simple model of circumstellar envelopes, Kozasa et al. (1996) have proposed that the $11.3 \mu \mathrm{m}$ feature could be attributed to small spherical core/mantle type grains (composed by a $\alpha$-SiC core and a carbon mantle) in most cases. In their calculations they have used optical constants for $\mathrm{SiC}$ tabulated by Choyke \& Palick (1985), which peak at about $10.7 \mu \mathrm{m}$. In fact, using this set of constants, the emission produced by a core/mantle grain as proposed by them is about $11.3 \mu \mathrm{m}$, and almost all sources of the SLMP sample could be fitted using a core/mantle grain code. This is no longer true when we use the constants proposed by Pégourié (1988), which peak at about $11.3 \mu \mathrm{m}$. In this case, the emission is shifted to longer wavelengths, too. We have computed our models considering the constants by Pégourié (1988) because the full treatment consisting of the Kramers-Kronig analysis has been taken into account.

Another possibility was raised by Speck et al. (1999): they have fitted some carbon stars using $\beta$-SiC grains. Silicon carbide grains can be divided into two basic groups: $\alpha-\mathrm{SiC}$ if the structure is one of the many hexagonal or rhombohedral polytypes, and $\beta$-SiC if the structure is cubic. $\beta$-SiC feature occurs at about $0.4 \mu \mathrm{m}$ shortwards of that of $\alpha$-SiC. Their results were obtained without the
$\mathrm{KBr}$ correction and they determined that $\alpha$-SiC has an intense, broad band near $11.8 \mu \mathrm{m}$ and $\beta$-SiC peaks at 11.3 to $11.4 \mu \mathrm{m}$. Silicon carbide grains found in meteorites have isotopic compositions that imply that most of these grains were formed around carbon stars. All studies to date of meteoritic SiC grains have found them to be of the $\beta$-type (Bernatowicz 1997). $\beta$-SiC will transform into $\alpha$-SiC above $2100{ }^{\circ} \mathrm{C}$ but the reverse process is thermodynamically unlikely. The results obtained by Speck et al. (1999) show that there is an obvious predominance of the $\beta$-SiC phase and that there is now no evidence for the $\alpha$-SiC phase at all. Their sample contains $\mathrm{SiC}, \mathrm{SiC}+$, Red and Extreme Carbon stars. However they do not solve the radiative transfer in these media. Moreover, we should expect some difference between "early" and "late" carbon stars with regard to dust grains. Unfortunately, the optical constants for $\beta$-SiC were calculated in a short range of wavelengths, about 7 to $12 \mu \mathrm{m}$. In this case, we need to adopt another set of optical constants at shorter wavelengths, where most of the stellar radiation is concentrated. With this assumption we cannot prove that $\beta$-SiC grains are responsible for the $11.3 \mu \mathrm{m}$ emission alone.

SLMP have suggested the following carbon-rich dust sequence: $\mathrm{SiC}+\rightarrow \mathrm{SiC} \rightarrow$ Red. The Red sources are significantly cooler on average than the $\mathrm{SiC}+$ sources. Following our results, $\mathrm{SiC}+$ stars have thinner envelopes than $\mathrm{SiC}$ stars. Temperatures of the central stars are very similar but there is a tendency to cooler temperatures in this sequence. Best models were obtained with two homogeneous grains for both $\mathrm{SiC}+$ and $\mathrm{SiC}$ class. Red stars were best described with core/mantle grains. As mentioned before, based on our results we suggest that our sample of extreme carbon stars contains $\mathrm{SiC}$ and Red stars. In this sample, the thinner envelopes were best represented by two homogeneous grains models (our E1 group) while thicker ones by core/mantle grain models (E2 group). The temperature of these stars are also cooler than in the SiC+ class. These results suggest that the sequence proposed by SLMP can be interpreted as an evolutionary scenario. Moreover, it seems reasonable to include our sample of extreme carbon stars in such a scenario. In the beginning of the sequence, grains are mainly composed of $\alpha$-SiC and amorphous carbon; with dust envelope evolution, carbon grains become coated $\alpha$-SiC ones. (Hence the emission is shifted to longer wavelengths). This phenomenon could perhaps explain the small quantities of $\mathrm{SiC}$ grains observed in the interstellar medium.

Concerning $\mathrm{SiC}++$ class stars, SLMP have proposed that they lie in a different evolutionary sequence, related to J-type carbon stars. In their sample, which contains 96 objects, only nine are J-type stars. Two of them are classed in the $\mathrm{SiC}++$ class. One of us (Lorenz-Martins 1996) have proposed that J-type carbon stars have an alternative evolutionary scenario which differs from that proposed for ordinary carbon stars. In fact, according to the results of the present paper, $\mathrm{SiC}++$ stars have thicker $(0.02 \leq \tau \leq$ $0.20)$ envelopes than those of J-type stars $(0.01 \leq \tau \leq$ 0.05, see Lorenz-Martins, 1996). It seems that some 
correlation between $\mathrm{SiC} / \mathrm{A}$.C. ratios of both groups of stars also exists. Such results reinforce the suggestion by SLMP linking $\mathrm{SiC}++$ and J-type carbon stars.

Acknowledgements. S. Lorenz Martins acknowledges FUJB (FUJB 8635-5) for financial support. We would like to thank Dr. R. Rabaça for his careful reading of the manuscript and also the referee, Dr. I. Little-Marenin for constructive comments and suggestions. This research was performed using the SIMBAD database at Strasbourg.

\section{References}

Aoki, W., Tsuji, T., \& Ohnaka, K. 1999, A\&A, 350, 945

Atlas of Low Resolution IRAS Spectra,1986, Science Team, ed. F. M. Olnon, \& E. Raimond, A\&AS, 65, 607

Bangulo, S. 1996, Ph.D. Thesis, The Queen's University of Belfast

Bagnulo, S., Doyle, J. G., \& Andretta, V. 1998, MNRAS, 296, 545

Beck, H. K. 1992, private communication

Bergeat, J., Sibille, F., Lunel, M., \& Lefèvre, J. 1976, A\&A, 52,227

Bergeat, J., \& Lunel, M. 1980, A\&A, 139, 141

Bernatowicz, T. 1997, in From stardust to planetesimal, ed. Y. Pendleton, \& A. G. G. M. Tielens, ASP Conf. Ser., 122, 227

Bohren, C. F., \& Huffman, D. R. 1998, in Absorption and scattering of light by small particles (John Wiley \& Sons)

Cernicharo, J., Guélin, M., \& Kahane, C. 2000, A\&AS, 142, 181

Chan, S. C., \& Kwok, S. 1990, A\&A, 237, 354

Choyke, W. J., \& Palik, E. D. 1985, in Handboook of Optical Constants of Solids, ed. E. D. Palik (Academic Press, Boston), 587

Cohen, M. 1984, MNRAS, 206, 137

Dischler, B., Bubenzer, A., \& Koidl, P. 1983, Appl. Phys. Lett., 42,636

Epchtein, N., Le Bertre, T., \& Lépine, J. R. D. 1990, A\&A, 227,104

Forrest, W. J., Gillett, F. C., \& Stein, W. A. 1975, ApJ, 195, 423

Fouqué, P., Le Bertre, T., Epchtein, N., Guglielmo, F., \& Kerschbaum, F. 1992, A\&AS, 93, 151

Gehrz, R. D., \& Hackwell, J. A. 1976, ApJ, 206, L161

Gezari, D. Y., Schmitz, M., \& Mead, J. M. 1987, Catalog of Infrared Observations, second edition, NASA RP-1196

Gilman, R. C. 1969, ApJ, 155, L185

Goebel, J. H., Cheeseman, P., \& Gerbault, F. 1995, ApJ, 449, 246

Griffin, J. P. 1990, MNRAS, 247, 591

Groenewegen, M. A. T. 1995, A\&A, 293, 463

Groenewegen, M. A. T. 1997, A\&A, 317, 503

Hackwell, J. A. 1972, A\&A, 21, 239
Hoyle, F., \& Wickramasinghe, N. C. 1991, The theory of cosmic grains (Kluwer Ac. Press)

Johnson, J. J., \& Jones, T. J. 1991, AJ, 101, 1735

Jones, T. J., Bryja, C. O., Gehrz, R. D., et al. 1990, ApJS, 74, 785

Kerschbaum, F., Lazaro, C., \& Habison, P. 1996, A\&AS, 118, 397

Kholopov, P. N., Samus, N. N., Frolov, M. S., et al. 1987, General Catalogue of Variable Stars, fourth edition, Nauka

Knapp, G. R., \& Morris, M. 1985, ApJ, 292, 640

Knapp, G. R., Dobrovolsky, S. I., Ivezić, Z., et al. 2000, A\&A, 351, 97

Kozasa, T., Dorschner, J., Henning, T., \& Stognienko, R. 1996, A\&A, 307, 551

Le Bertre, T. 1987, A\&A, 176, 107

Le Bertre, T. 1988, A\&A, 190, 79

Le Bertre, T. 1992, A\&AS, 94, 377

Little-Marenin, I. E., Ramsey, M. E., Stephenson, C. B., Little, S. J., \& Priece, S. D. 1987, AJ, 93, 663

Lorenz-Martins, S., \& Lefèvre, J. 1993, A\&A, 280, 567

Lorenz-Martins, S., \& Lefèvre, J. 1994, A\&A, 291, 831

Lorenz-Martins, S. 1996, A\&A, 314, 209

Lorenz-Martins, S., \& Araújo, F. X. 1997, MNRAS, 291, 296

Martin, P. G., \& Rogers, C. 1987, ApJ, 322, 374

Mauron, N., \& Huggins, P. J. 1999, A\&A, 349, 203

McCabe, E. M. 1982, MNRAS, 200, 71

Mitchell, R. M., \& Robinson, G. 1980, MNRAS, 190, 669

Noguchi, K., Kawara, K., et al. 1981, PASJ, 33, 373

Noguchi, K., \& Akiba, M. 1986, PASJ, 38, 811

Nyman, L. - A., Booth, R. S., Carlström, U., et al. 1992, A\&AS, 93, 121

Olofsson, H., Eriksson, K., \& Gustafsson, B. 1990, A\&A, 230, 405

Papuolar, R. 1988, A\&A, 204, 138

Pégourié, B. 1988, A\&A, 194, 335

Rouleau, F., \& Martin, P. G. 1991, ApJ, 377, 526

Rowan-Robinson, M., \& Harris, S. 1983, MNRAS, 202, 797

Speck, A. K., Hofmeister, A. M., \& Barlow, M. J. 1999, ApJ, 513, L87

Sloan, G. C., Little-Marenin, I. R., \& Price, S. D. 1998, AJ, 115,809

Suh, K.-W. 2000, MNRAS, 315, 740

Trammell, S. R., Dinerstein, H. L., \& Goordrich, R. W. 1994, AJ, 108, 984

Treffers, R., \& Cohen, M. 1974, ApJ, 188, 545

Walker, A. 1976, MNRAS, 174, 609

Whittet, D. C. B., Duley, W. W., \& Martin, P. G. 1990, MNRAS, 174, 609

Willems, F. J. 1987, A\&A, 203, 65

Yamamura, I., de Jong, T., Walters, L. B. F. M., \& Cami, J. 1998, in Asymptotic Giant Branch Stars, ed. T. Le Bertre, A. Lèbre, C. Waelkens

Yamashita, Y. 1972, Tokyo Astron. Obs. Ann., 13, 169

Zubko, V. 1996, MNRAS, 282, 1321 